\title{
QUALIDADE DE VIDA E TRANSTORNOS MENTAIS COMUNS EM ESTUDANTES DE MEDICINA
}

\author{
Lais Silva dos Santos ${ }^{1}$, Ícaro José Santos Ribeiro², Eduardo Nagib Boery ${ }^{3}$, Rita Narriman Silva de Oliveira \\ Boery $^{4}$
}

RESUMO: Objetivou-se avaliar a associação entre a qualidade de vida e transtornos mentais comuns em estudantes de medicina da Universidade Estadual do Sudoeste da Bahia campus de Jequié. Estudo de corte transversal desenvolvido com 115 estudantes (61 homens e 54 mulheres) durante o segundo semestre de 2016. A prevalência de transtornos mentais comuns foi de $32,2 \%(n=37)$. O menor domínio da qualidade de vida entre os indivíduos com transtornos mentais comuns foi o meio ambiente com mediana de 56,4 (IQ 46,9-68,8), seguido pelo psicológico 56,9 (IQ 50-66,6); físico 57,1 (IQ 46,6-67,8); e relações sociais 65,5 (IQ 50-83,3). Vislumbra-se que a totalidade dos domínios da qualidade de vida foram reduzidos em indivíduos com suspeição de transtornos mentais comuns, tendo o modelo final da regressão evidenciado os domínios físico e psicológico como fator de proteção para os mesmos.

DESCRITORES: Qualidade de vida; Saúde mental; Estudantes; Transtornos Mentais; Epidemiologia.

\section{QUALITY OF LIFE AND COMMON MENTAL DISORDERS AMONG MEDICAL STUDENTS}

ABSTRACT: The aim of this study was to evaluate the association between quality of life and common mental disorders among medical students from the State University of Southwest Bahia, Jequié campus. A cross-sectional study was conducted with 115 students (61 male and 54 female students) during the second half of 2016 . The prevalence of common mental disorders was $32.2 \%$ $(\mathrm{n}=37)$. The lowest domain of quality of life among individuals with common mental disorders was the environment, with median of 56.4 (IQ 46.9-68.8), followed by psychological, 56.9 (IQ 50-66.6); physical health, 57.1 (IQ 46.6-67.8); and social relations, 65.5 (IQ 50 83.3). This study estimates that all domains of quality of life were reduced in individuals with suspected common mental disorders; and the final regression model showed the physical health and psychological domains as a protection factor for these individuals. DESCRIPTORS: Quality of life; Mental health; Students; Mental disorders; Epidemiology.

\section{CALIDAD DE VIDA Y TRASTORNOS MENTALES COMUNES EN ESTUDIANTES DE MEDICINA}

RESUMEN: Se objetivó evaluar la asociación entre calidad de vida y trastornos mentales comunes en estudiantes de medicina de la Universidade Estadual do Sudoeste da Bahia, campus de Jequié. Estudio de corte transversal desarrollado con 115 estudiantes (61 hombres y 54 mujeres) durante el segundo trimestre de 2016. La prevalencia de trastornos mentales comunes fue del $32,2 \%$ ( $\mathrm{n}=37$ ). El menor dominio de calidad de vida entre los individuos con trastornos mentales comunes fue medio ambiente, con mediana de 56,4 (IQ 46,9-68,8), seguido del psicológico 56,9 (IQ 50-66,6); físico 57,1 (IQ 46,6-67,8); y relaciones sociales 65,5 (IQ 50-83,3). Se observa que la totalidad de dominios de calidad de vida fueron reducidos en individuos con sospecha de trastornos mentales comunes, habiendo el modelo final de la regresión evidenciado los dominios físico y psicológico como factor de protección para los mismos. DESCRIPTORES: Calidad de Vida; Salud Mental; Estudiantes; Trastornos Mentales; Epidemiología.

1Discente de em Enfermagem. Universidade Estadual do Sudoeste da Bahia. Jequié, BA, Brasil.

${ }^{2}$ Enfermeiro. Mestre em Ciências. Discente do Programa de Pós-graduação em Enfermagem e Saúde da Universidade Estadual do Sudoeste da Bahia. Jequié, BA, Brasil.

${ }^{3}$ Enfermeiro. Doutor em Enfermagem. Docente da Universidade Estadual do Sudoeste da Bahia. Jequié, BA, Brasil.

${ }^{4}$ Enfermeira. Pós-Doutora em Bioética. Docente da Universidade Estadual do Sudoeste da Bahia. Jequié, BA, Brasil.

Autor Correspondente:

Ícaro José Santos Ribeiro

Universidade Estadual do Sudoeste da Bahia

Av. José Moreira Sobrinho, s/n - 45.206-510 - Jequié, BA, Brasil

E-mail: icaro.ribeiro29@gmail.com
Recebido: 02/05/2017

Finalizado: 31/10/2017 


\section{INTRODUÇÃO}

Os fatores estressores inerentes aos processos formativos na educação médica são potenciais desencadeadores de transtornos mentais, entre eles os transtornos mentais comuns (TMC). As consequências da instalação desses quadros transcendem as manifestações clínicas, podendo causar deterioração da qualidade de vida dos estudantes.

Os transtornos mentais comuns são grupos menos graves e mais frequentes de transtornos mentais, sendo que os sintomas incluem esquecimento, dificuldade na concentração, insônia, irritabilidade e fadiga, assim como queixas somáticas (i.e. cefaleia, falta de apetite, tremores, má digestão) ${ }^{(1-2)}$.

Os cursos de graduação na área de saúde (e.g. Enfermagem, Fisioterapia, Medicina), em virtude de sua carga horária de período integral, requerem maior dedicação e esforço dos alunos do curso, podendo acarretar em comprometimento da vida social e do bem-estar físico, afetando assim a Qualidade de Vida (QV) desses estudantes ${ }^{(3)}$. A QV é definida como a percepção do indivíduo em relação ao contexto em que se encontra e desenvolve suas atividades, bem como seus objetivos, preocupações e expectativas. Trata-se de um conceito amplo em que pode ser avaliado o estado psíquico, físico, relações sociais e características ambientais em que o mesmo se acha inserido ${ }^{(4)}$.

O estado de estresse vem sendo apontado como frequente entre estudantes, que, por sua vez, conservam essa condição após a vida acadêmica. Além disso, estudos apontam o uso de drogas e a alta prevalência de suicídio, distúrbios psicológicos e conjugais entre essa população, podendo acarretar prejuízos não só a si, como também ao paciente, que passa a ter o cuidado negligenciado(4).

Dessa forma, a avaliação de questões relacionadas às condições de saúde e QV de estudantes de medicina viabilizará o entendimento de aspectos implicados nessa relação e subsidiará ações voltadas à prevenção e cuidados para este público ${ }^{(5)}$.

Assim, este estudo objetivou avaliar a associação entre a qualidade de vida e TMC entre os estudantes de medicina da Universidade Estadual do Sudoeste da Bahia, campus de Jequié.

\section{MÉTODO}

Trata-se de um estudo epidemiológico analítico, de corte transversal. Foram informantes desta pesquisa 115 estudantes (61 homens e 54 mulheres) regularmente matriculados no curso de medicina da Universidade Estadual do Sudoeste da Bahia, campus de Jequié. Os dados foram obtidos durante o primeiro e segundo semestres de 2016. Foram incluídos estudantes regularmente matriculados, maiores de 18 anos e que consentiram em participar. Foram excluídos os graduandos de medicina que estavam cursando matéria optativa fora do campus de Jequié.

Para fins de análise, a suspeição de TMC foi utilizada como variável dependente, enquanto a QV como variável independente principal. Outras variáveis foram incluídas como independentes, sendo elas sexo, raça/cor, situação marital e situação de moradia (mora sozinho e mora em república).

A coleta de dados referente à QV foi realizada mediante aplicação do WHOQOL-BREF, instrumento utilizado para avaliar a qualidade de vida de populações adultas, constando de 26 questões distribuídas em quatro domínios: físico, psicológico, relações sociais e meio ambiente ${ }^{(6)}$. A suspeição de TMC foi mensurada por meio do Self Reporting Questionnaire (SRQ-20), instrumento multidimensional contendo 20 perguntas com respostas binárias (sim ou não) que contemplam grupo de sintomas como humor depressivo-ansioso, sintomas somáticos, decréscimo de energia vital, pensamentos depressivos $^{(7)}$.

No que se refere à classificação de suspeição de TMC, destaca-se a existência de variações nos pontos de corte adotados para suspeição diagnóstica da morbidade psíquica. Dessa forma, optou-se por utilizar o critério que considera que pontuações $\leq 6$ representam não suspeição de TMC e $>6 \mathrm{com}$ suspeição de $\mathrm{TMC}^{(7)}$.

Os dados foram avaliados por estatística descritiva. Foram construídas as distribuições de 
frequências (relativa e absoluta) e calculadas as medianas e intervalos interquartil de cada variável, pela característica não paramétrica das variáveis estudadas (i.e. Kolmogorov Smirnov < 0,05).

Para fins de comparação das medianas dos domínios da QV de acordo com a suspeição ou não de TMC, foi utilizado o teste de Mann Whitney. Posteriormente, um modelo de regressão logística foi aplicado considerando as variáveis que apresentaram valor de $p<0,2$ na comparação das medianas. As associações significantes no modelo multivariado foram expressas pelo coeficiente beta e seu respectivo intervalo de confiança. Todas as análises foram realizadas pelo Statistical Package for the Social Sciences (SPSS) versão 21.0.

A pesquisa foi aprovada em maio de 2013, pelo do Comitê de Ética e Pesquisa da Universidade Estadual do Sudoeste da Bahia (UESB), parecer 274.134.

\section{RESULTADOS}

Foram entrevistados 115 estudantes do curso de medicina (61 homens e 54 mulheres) com média de idade de 24,7 anos $( \pm 4,8)$. A prevalência de transtornos mentais comuns foi de $32,2 \%(n=37)$. A Tabela 1 evidencia as características da população estudada de acordo com a suspeição de TMC. Assim, entre os indivíduos com suspeição de transtorno mental comum, $70,3 \%(n=26)$ eram do sexo feminino; $48,6 \%$ $(\mathrm{n}=18)$ da cor parda; 64,9\% ( $\mathrm{n}=24)$ possuíam companheiro; e 75,0\% ( $\mathrm{n}=27)$ não moravam sozinhos.

Tabela 1 - Características da população estudada segundo a suspeição de transtorno mental comum. Jequié, BA, Brasil, 2016

\begin{tabular}{lcc} 
& \multicolumn{2}{c}{ Suspeição de TMC } \\
\cline { 2 - 3 } & Não [n (\%)] & Sim [n (\%)] \\
\hline Sexo & $28(35,9)$ & $26(70,3)$ \\
\hline Feminino & $50(64,1)$ & $11(29,7)$ \\
\hline Masculino & & \\
\hline Raça/Cor & $24(30,8)$ & $13(35,1)$ \\
\hline Branca & $35(44,9)$ & $18(48,6)$ \\
\hline Parda & $10(12,8)$ & $55(13,5)$ \\
\hline Preta & $5(6,4)$ & $0(0)$ \\
\hline Indígena & & $13(35,1)$ \\
\hline Situação Marital & $39(51,3)$ & $24(64,9)$ \\
\hline Sem companheiro & $37(48,7)$ & $9(25)$ \\
\hline Com companheiro & & $27(75)$ \\
\hline Mora sozinho & $24(30,8)$ & \\
\hline Sim & $54(69,2)$ & \\
\hline Não & & \\
\hline
\end{tabular}

Na Tabela 2, observam- se variáveis referentes à qualidade de vida. Vislumbra-se que a totalidade dos domínios da QV são reduzidos em indivíduos com suspeição de TMC. O menor domínio da QV entre os indivíduos com TMC foi o meio ambiente com mediana de 56,4 (IQ 46,9-68,8), seguido pelo psicológico 56,9 (IQ 50-66,6); físico 57,1 (IQ 46,6-67,8); e relações sociais 65,5 (IQ 50-83,3). Tal diferença entre as medianas evidenciadas mostrou-se estatisticamente significativa pelo teste de Mann Whitney. 
Tabela 2 - Distribuição dos domínios da qualidade de vida e suspeição de transtornos mental comum. Jequié, BA, Brasil, 2016

\begin{tabular}{lccccc} 
& \multicolumn{2}{c}{ Sem suspeição } & \multicolumn{2}{c}{ Com suspeição } & \multirow{2}{*}{ P } \\
\cline { 2 - 5 } & Mediana & IQ & Mediana & IQ & \\
\hline Físico & 76,8 & $67,8-85,7$ & 57,1 & $46,6-67,8$ & $<0,05$ \\
\hline Psicológico & 74,2 & $66,6-83,3$ & 56,9 & $50,0-66,6$ & $<0,05$ \\
\hline Relações sociais & 77 & $75,0-91,6$ & 65,5 & $50,0-83,3$ & $<0,05$ \\
\hline Meio ambiente & 65 & $56,2-75$ & 56,4 & $46,9-68,8$ & $<0,05$ \\
\hline Índice geral & 71,4 & $62,5-75$ & 60,4 & $50-75$ & $<0,05$
\end{tabular}

O modelo final da regressão logística está apresentado na Tabela 3, sendo observados os coeficientes de regressão $\beta$ de cada variável que permaneceu no modelo, assim como o coeficiente ajustado $\beta$. É possível notar que os coeficientes foram atenuados, porém o domínio físico e o psicológico permaneceram associados à suspeição de TMC.

Tabela 3 - Coeficiente de regressão $\beta$ com e sem ajuste, e intervalo de confiança 95\% de modelo final de regressão para risco de TMC. Jequié, BA, Brasil, 2016

\begin{tabular}{lcccc} 
& $\boldsymbol{\beta}$ & IC95\% & $\boldsymbol{\beta}$ ajustado & IC95\% para $\boldsymbol{\beta}$ ajustado \\
\hline Domínio Físico & 0,918 & $0,875-0,964$ & 0,929 & $0,892-0,969$ \\
\hline Domínio Psicológico & 0,942 & $0,899-0,986$ & 0,949 & $0,911-0,989$
\end{tabular}

\section{- DISCUSSÃO}

Esse estudo evidenciou maior prevalência de TMC em indivíduos do sexo feminino, brancos, com companheiro e que não moravam sozinhos, nem em república. Quanto à qualidade de vida, todos os domínios foram diminuídos em indivíduos com suspeição de TMC.

Estudos acerca da prevalência de TMC são realizados em diferentes populações por todo o país. Observando achados de outras pesquisas com estudantes de medicina no Brasil, verificou-se que a prevalência de TMC aqui evidenciada (32,2\%) foi menor do que os resultados de estudos realizados no Espírito Santo $(37,1 \%)^{(8)}$, Pernambuco $(42,6 \%)^{(9)}$ e São Paulo $(44,7 \%)^{(10)}$. Por outro lado, mostrou-se semelhante, menor ou ligeiramente superior aos achados de Bahia $(29,6 \%)^{(11)}$, Sergipe $(33,3 \%)^{(12)}$ e Paraíba $(33,6 \%)^{(13)}$.

Os diferentes resultados evidenciados, no que concerne a prevalência de TMC nos diferentes estudos, podem estar relacionados às diferenças no processo ensino-aprendizagem, estrutura curricular, bem como diferenças regionais ou metodológicas relativas aos delineamentos utilizados ${ }^{(14)}$.

O estudo apontou a maior prevalência de TMC em estudantes do sexo feminino (70,3\%). De forma similar, estudos observaram maior acometimento por TMC em graduandas de medicina do sexo feminino, oscilando de 40,0 a $88,0 \%{ }^{(8,15-17)}$.

Graduandos do sexo feminino, quando comparados aos do sexo masculino, possuem dificuldade em conciliar o internato com os estudos, ou seja, dificuldade com dedicação no período integral. Possíveis causas desse risco aumentado em mulheres são interações entre influências hormonais, diferenças sexuais ligadas a aspectos neuronais envolvidas com humor e ansiedade, estressores psicossociais, papéis de gênero prescritos e a importância do apoio social para mulheres ${ }^{(18-19)}$.

Viver longe dos familiares é outro fator influenciador da QV e que propicia nos primeiros 
meses isolamento social, por ser uma fase de integração com um novo estilo vida e aparecimento de sentimentos, como saudade e tristeza. Com isso, a dimensão psicológica pode ser afetada, o que justifica a ocorrência comum de transtornos mentais, bem como o grande índice de suicídio e depressão nessa população ${ }^{(4,8)}$.

A formação médica parece ter um impacto negativo sobre a saúde mental dos alunos, podendo haver vários determinantes dessa situação, como a sobrecarga acadêmica ${ }^{(20-21)}$, frequências elevadas de estresse $^{(22-23)}$, ansiedade e depressão ${ }^{(24-26)}$ e relação destes com menores valores da qualidade de vida.

A grande demanda dos graduandos de medicina gera forte pressão e estresse pela exigência de alto rendimento, que reduz a quantidade de sono, levando-os a ficarem acordados por mais tempo, a fim de atender às exigências acadêmicas para compreender amplos conteúdos do exercício no campo prático. Como resultado disso, ocorre alteração do ciclo do sono, visto que a insônia, apresentada pela fragmentação do sono, promove no indivíduo o cansaço e a sonolência durante o dia, comprometendo seu desempenho e proporcionando o desgaste físico e psíquico ${ }^{(27)}$.

Considerando-se a avaliação feita pelo SRQ-20, percebe-se a necessidade de os estudantes passarem por uma avaliação do profissional de saúde mental, uma vez que a QV está notadamente afetada, principalmente os aspectos de relações sociais e psicológico. Esses são talvez os domínios mais influenciados no desenvolvimento de transtornos mentais comuns, já que o estresse, evidenciado frequentemente em graduandos de medicina, é cada vez mais preocupante, pois pode acarretar alterações fisiológicas, doenças cardiovasculares e desequilíbrio na atuação do sistema nervoso, os quais desencadeiam o declínio da QV por gerar irritação, impaciência, depressão, infelicidade no âmbito pessoal, alterando, por fim, o jeito de ser do indivíduo ${ }^{(18)}$.

A relação dos TMC com a qualidade de vida também foi mensurada em pesquisa com 1.762 jovens, de 18 a 24 anos, da cidade de Pelotas-RS. Evidenciou-se que todos os domínios da QV mostraram associações significativas com TMC, sendo que as pessoas com TMC obtiveram menores escores quando comparadas com aquelas sem $\mathrm{TMC}^{(28)}$.

As instituições de ensino superior devem refletir acerca das necessidades e condições de saúde que o ensino médico promove, conhecendo as características dos seus alunos e os momentos considerados críticos ao decorrer do curso, como forma de estipular estratégias para contribuir com os discentes. A importância da saúde mental é reconhecida pela Organização Mundial de Saúde quando é definido o conceito de saúde enfocando para o estado mental, assim como o físico e o social ${ }^{(8)}$.

A mudança na QV dos graduandos de Medicina passa por mudanças de postura dos próprios indivíduos, desempenhando sua personalidade para as situações adversas decorrentes do seu curso ${ }^{(29)}$. A transição da escola para o ensino superior está associada ao aumento da incidência de problemas de saúde mental, devido aos múltiplos estressores e mudanças de estilo de vida envolvidos ${ }^{(30)}$.

O fato de o domínio físico e psicológico apresentar-se como fator de proteção para o desenvolvimento de TMC parece estar intimamente relacionado ao conteúdo interno avaliado por cada um desses domínios. O domínio físico avalia questões relacionadas à dor e desconforto, energia e fadiga, atividade sexual, sono e repouso e funções motoras; o psicológico avalia sentimentos positivos e negativos, memória e concentração e autoestima ${ }^{(31)}$.

Assim, o incremento dos valores obtidos nesses domínios representará, consequentemente, maior energia e disposição, melhores padrões de sono e repouso, melhor memória e concentração, autoestima, etc. Tais elevações estão relacionadas à melhora nas condições de saúde mental e na redução dos transtornos mentais comuns ${ }^{(28)}$.

Salienta-se que apesar dos resultados aqui evidenciados, o desenho metodológico adotado, corte transversal, impossibilita a afirmação de relação causal entre as variáveis estudadas. Todavia, por abordar a totalidade dos estudantes de medicina da instituição em questão, apresenta um retrato real da situação de saúde e o risco aos quais estão expostos estes indivíduos.

\section{- CONCLUSÃO}


Os resultados demonstram que os domínios físico e psicológico da qualidade de vida são fatores de proteção para o desenvolvimento de transtornos mentais comuns em estudantes de medicina. Dessa forma, urge a adoção de medidas que incrementem tais domínios, como a prática de atividade física e de lazer, para que os TMC não sejam depressores da saúde geral dos estudantes e possam agravar outros aspectos das suas vidas.

Salienta-se que a formação em saúde, não exclusivamente médica, é fator predisponente para o adoecimento mental e redução da qualidade de vida, dados os fatores aqui discutidos (i.e. carga horária excessiva, convívio com sofrimento humano, responsabilidades etc.). Assim, novas pesquisas com discentes das diferentes áreas (i.e. Enfermagem, Fisioterapia etc.) poderão propor abordagens preventivas melhor direcionadas e resolutivas.

\section{REFERÊNCIAS}

1. Tófoli LF. Transtornos somatoformes, síndromes funcionais e sintomas físicos sem explicação médica. In: Tratado de clínica médica. 1a ed. São Paulo: Roca; 2006.

2. Goldberg D. A bio-social model for common mental disorders. Acta Psychiatr Scand Suppl. 1994;385:66-70.

3. Olmo NRS, Ferreira LF, Prado AD, Martins LC, Dedivitis RA. Percepção dos estudantes de medicina do primeiro e sexto anos quanto à qualidade de vida. Diagn Tratamento. 2012;17(4):157-61.

4. Alves JGB, Tenório M, dos Anjos AG, Figueroa JN. Qualidade de vida em estudantes de Medicina no início e final do curso: avaliação pelo Whoqol-bref. Rev. bras. educ. med. 2010;34(1):91-6.

5. Chazan ACS, Campos MR. Qualidade de vida de estudantes de medicina medida pelo WHOQOL-bref - UERJ, 2010. Rev. bras. educ. med. 2013;37(3):376-84.

6. Fleck MPA, Louzada S, Xavier M, Chachamovich E, Vieira G, Santos L, et al. Aplicação da versão em português do instrumento abreviado de avaliação da qualidade de vida "WHOQOL-bref." Rev. Saúde Pública. 2000;34(2):17883.

7. Santos KOB, de Araújo TM, Pinho PS, Silva ACC. Avaliação de um instrumento de mensuração de morbidade psíquica: estudo de validação do self-reporting questionnaire (srq-20). Rev Baiana Saúde Pública. 2010;34(3):54460.

8. Fiorotti KP, Rossoni RR, Borges LH, Miranda AE. Transtornos mentais comuns entre os estudantes do curso de medicina: prevalência e fatores associados. J. bras. psiquiatr. 2010;59(1):17-23.

9. Facundes VLD, Ludermir AB. Common mental disorders among health care students. Rev. Bras. Psiquiatr. 2005;27(3):194-200.

10. Lima MCP, Domingues MS, Cerqueira ATAR. Prevalência e fatores de risco para transtornos mentais comuns entre estudantes de medicina. Rev. Saúde Pública. 2006;40(6):1035-41.

11. Almeida AM, Godinho TM, Bitencourt AGV, Teles MS, Silva AS, Fonseca DC, et al. Common mental disorders among medical students. J. Bras. Psiquiatr. 2007;56(4):245-51.

12. Costa EFO, de Andrade TM, Silvany Neto AM, de Melo EV, Rosa ACA, Alencar MA, et al. Common mental disorders among medical students at Universidade Federal de Sergipe: a cross-sectional study. Rev. Bras. Psiquiatr. 2010;32(1):11-9.

13. Rocha ES, Sassi AP. Minor mental disorders among medical students. Rev. bras. educ. med. 2013;37(2):210-6.

14. Ferreira CMG, Kluthcovsky ACGC, Cordeiro TMG. Prevalência de transtornos mentais comuns e fatores associados em estudantes de medicina: um estudo comparativo. Rev. bras. educ. med. 2016;40(2):268-77.

15. Coutinho LMS, Matijasevich A, Scazufca M, Menezes PR. Prevalência de transtornos mentais comuns e contexto social: análise multinível do São Paulo Ageing \& Health Study (SPAH). Cad. Saúde Pública. 2014;30(9):1875-83. 
16. Silva RS, da Costa LA. Prevalência de transtornos mentais comuns entre estudantes universitários da área da saúde. Encon.: R. Psicol. 2012;15(23):105-12.

17. Padovani RC, Neufeld CB, Maltoni J, Barbosa LNF, de Souza WF, Cavalcanti HAF, et al. Vulnerability and psychological well-being of college student. Rev Bras Ter Cogn. 2014;10(1):2-10.

18. Meyer C, Guimarães ACA, Machado Z, Parcias SR. Qualidade de vida e estresse ocupacional em estudantes de medicina. Rev. bras. educ. med. 2012;36(4):489-98.

19. King SL, Hegadoren KM. An integrative science approach: Value added in stress research. Nurs Heal Sci. 2006;8(2):114-9.

20. Sadir MA, Bignotto MM, Lipp MEN. Stress e qualidade de vida: influência de algumas variáveis pessoais. Paidéia (Ribeirão Preto). 2010;20(45):73-81.

21. de Vasconcelos TC, Dias BRT, Andrade LR, Melo GF, Barbosa L, Souza E. Prevalência de sintomas de ansiedade e depressão em estudantes de medicina. Rev. bras. educ. med.. 2015;39(1):135-42.

22. Abdulghani HM, AIKanhal AA, Mahmoud ES, Ponnamperuma GG, Alfaris EA. Stress and its effects on medical students: a cross-sectional study at a college of medicine in Saudi Arabia. J Health Popul Nutr. 2011;29(5):516-22.

23. Nechita F, Nechita D, Pirlog MC, Rogoveanu I. Stress in medical students. Rom J Morphol Embryol. 2014;55(3 Suppl):1263-6.

24. Domantay JAA. Health-related quality of life of future physicians at a medical school in the Philippines: a cross-sectional study. SAGE Open. 2014;4(3):1-9.

25. Kulsoom B, Afsar NA. Stress, anxiety, and depression among medical students in a multiethnic setting. Neuropsychiatr Dis Treat. 2015;(11):1713-22.

26. Rubin R. Recent suicides highlight need to address depression in medical students and residents. JAMA. 2014;312(17):1725-7.

27. Ribeiro CRF, da Silva YMGP, de Oliveira SMC. O impacto da qualidade do sono na formação médica. Rev Bras Clin Med. 2014;12(1):8-14.

28. Jansen K, Mondin TC, Ores LC, Souza LDM, Konradt CE, Pinheiro RT, et al. Transtornos mentais comuns e qualidade de vida em jovens: uma amostra populacional de Pelotas, Rio Grande do Sul, Brasil. Cad. Saúde Pública. 2011;27(3):440-8.

29. Bampi LNS, Baraldi S, Guilhem D, de Araújo MP, Campos ACO. Qualidade de vida de estudantes de Medicina da Universidade de Brasília. Rev. bras. educ. med. 2013;37(2):217-25.

30. Musiat P, Conrod P, Treasure J, Tylee A, Williams C, Schmidt U. Targeted prevention of common mental health disorders in university students: randomised controlled trial of a transdiagnostic trait-focused web-based intervention. PLoS One. 2014;9(4):e93621.

31. The WHOQOL Group. The World Health Organization Quality of Life Assessment (WHOQOL): development and general psychometric properties. Soc Sci Med. 1998;46(12):1569-85. 